\title{
EXPECTED BEAM PERFORMANCE OF THE SNS LINAC*
}

\author{
J. Stovall, J. H. Billen, S. Nath, H. Takeda, L. M. Young, Los Alamos National Laboratory, \\ D. Jeon, Oak Ridge National Laboratory, K. R. Crandall, R. Shafer, TechSource Inc.
}

\section{Abstract}

The Spallation Neutron Source (SNS) project is a collaboration among Argonne, Brookhaven, Jefferson, Lawrence Berkeley, Los Alamos and Oak Ridge National Laboratories. The Linac, which injects beam into an accumulator ring, is comprised of both normal and superconducting rf (SRF) accelerating structures. Two room-temperature $\mathrm{rf}$ structures, a $402.5-\mathrm{MHz}$ drift-tube linac (DTL) and an 805-MHz coupled-cavity linac (CCL), accelerate an H-minus beam from $2.5 \mathrm{MeV}$ to $186 \mathrm{MeV}$. The SRF linac accelerates the beam to $1 \mathrm{GeV}$ through 81 elliptical, multicell niobium cavities. This paper reviews the linac physics design and its expected beam dynamics performance.

\section{INTRODUCTION}

A previous paper [1], described the baseline design for a room-temperature $1-\mathrm{GeV}$ linac that would deliver 1.1 MW of average beam power for injection into the accumulator ring. In 2000, the baseline was modified and a substantial part of the CCL was replaced with superconducting cavities. This new linac was designed to deliver 2.65 MW of beam at $1.25 \mathrm{GeV}$. These performance requirements were subsequently modified three more times in an effort to keep the project within budget. The present linac is now designed to deliver 1.5 MW of beam at $1 \mathrm{GeV}$. In addition, the expected accelerating field in $60 \%$ of the SRF cavities has been increased by $27 \%$. In the meantime however, rf power system components, whose specifications were based on the requirements of previous designs, have been ordered, thus limiting our flexibility to optimize the "final" design.

Some of the linac design requirements and constraints are summarized in Table 1 . The most challenging requirements are to limit the beam spill and emittance growth.

Table 1: Linac design requirements and constraints

\begin{tabular}{ll}
\hline Parameter & Value \\
\hline $\mathrm{W}_{\text {initial }}$ & $2.5 \mathrm{MeV}$ \\
$\mathrm{W}_{\text {final }}$ & $1 \mathrm{GeV}$ \\
$\mathrm{I}_{\text {ave }}$ & $1.56 \mathrm{~mA}$ \\
$\mathrm{~W}_{\text {final }}$ stability & $\pm 1.5 \mathrm{MeV}$ \\
$\mathrm{W}_{\text {final }}$ spread & $\pm 0.33 \mathrm{MeV} \mathrm{rms}$ \\
$\varepsilon_{\text {initial, rms,norm }}$ & $\approx 0.27 \pi \mathrm{mm}$-mrad \\
$\varepsilon_{\text {final, rms,norm }}$ & $\leq 0.5 \pi \mathrm{mm}$-mrad \\
Beam loss & $\leq 1 \mathrm{~W} / \mathrm{m}$ throughout \\
Beam duty factor & $6 \%$ \\
Chopper duty & $68 \%$ \\
SRF cavities & $33 \mathrm{ea} \beta=.61,48$ ea $\beta=.81$ \\
$\mathrm{P}_{\text {klystron }}($ SRF $)$ & $\leq 550 \mathrm{~kW}$ \\
\hline
\end{tabular}

\section{DRIFT TUBE LINAC}

The DTL is comprised of $6 \mathrm{rf}$ tanks each powered by a single 2.5-MW klystron at $402.5 \mathrm{MHz}$. Two thirds of its 210 drift tubes contain permanent magnet quadrupole lenses (PMQs) arranged in a FFODDO lattice period of length $6 \beta \lambda_{402.5}$. The period lengths of the focusing lattices in the DTL and the following CCL are nearly equal, helping to achieve a smooth and nearly currentindependent match. Every third drift tube is available for either beam-position monitors (BPMs) or dipole steering magnets.

The DTL contains 24 electromagnetic dipoles and 10 BPMs that will also detect beam phase. Six beam current monitors (BCMs) will be located between tanks or in empty end-wall drift tubes. Additional diagnostics, located between tanks include wire-scanner beam profile monitors and energy-degrader/Faraday-cup assemblies. Ionization-type beam loss monitors are located throughout the linac tunnel.

The PMQs have all been delivered and have the same strength; $B^{\prime} \cdot L=13 \pm 0.5 \% \mathrm{kG}$. Drift spaces of length $1-\beta \lambda$ between tanks each removes one longitudinal focusing gap and the associated transverse defocusing lens in an otherwise uniform lattice. Uncompensated, these missing elements are a source of mismatch that cause significant halo development.
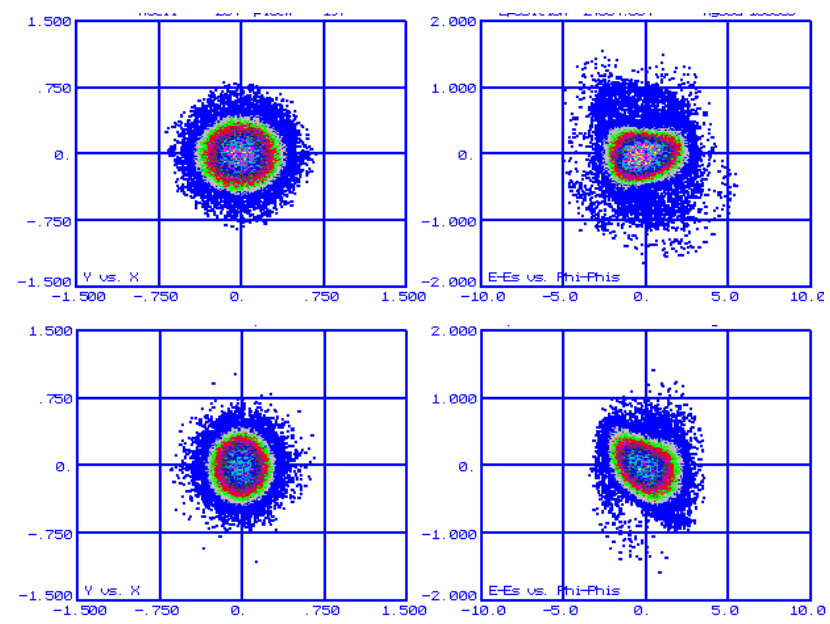

Figure 1. X-Y and $\mathrm{W}-\phi$ beam projections from PARMILA simulations using $10^{5}$ particles at $1 \mathrm{GeV}$ without (upper) and with (lower) DTL inter-tank matching.

To compensate for these missing elements we adjust the synchronous phase in the 6 adjacent gaps by about $\pm 3^{\circ}$ per cell by adjusting the cell lengths. These adjustments result in rf structures that are easy to tune and smooth the

*Work supported by the Office of Energy Research, Basic Energy Science of the US Department of Energy, and by Oak Ridge National Laboratory. 
average real-estate transverse and longitudinal phase advances $\left(\mathrm{k}_{0 \mathrm{t}}\right.$ and $\left.\mathrm{k}_{01}\right)$. The beneficial effect of this intertank matching is significant as one can see from the projections of the beam distribution in Fig. 1.

While there are multiple "knobs" available to match the beam into the DTL, there is no provision for matching the beam from tank to tank. If, in the future, it becomes apparent that additional transverse tuning of the lattice is required, space is reserved in otherwise unused empty drift tubes for electromagnetic trim quads having $\sim 10 \%$ of the nominal PMQ gradients.

To steer the beam through the DTL, we will use pairs of bipolar electromagnetic dipoles in tank $\mathrm{N}$ to steer the beam onto the axis of two BPMs located $\sim 90^{\circ}$ apart in tank $\mathrm{N}+1$. During this exercise tank $\mathrm{N}+1$ will be unexcited to assure that the BPMs are unaffected by the cavity rf fields.

We plan to use three types of "phase scans" to identify the correct rf phase and amplitude set points. Figure 2 shows the longitudinal acceptance of tank 3 for 3 different rf amplitudes. Figure 3 shows the traditional phase scan in which the initial beam, having the design energy, is scanned across the phase acceptance while measuring the accelerated component of the beam at the tank exit. The multiple curves represent different $\mathrm{rf}$ field amplitudes from $84-110 \%$ of design.

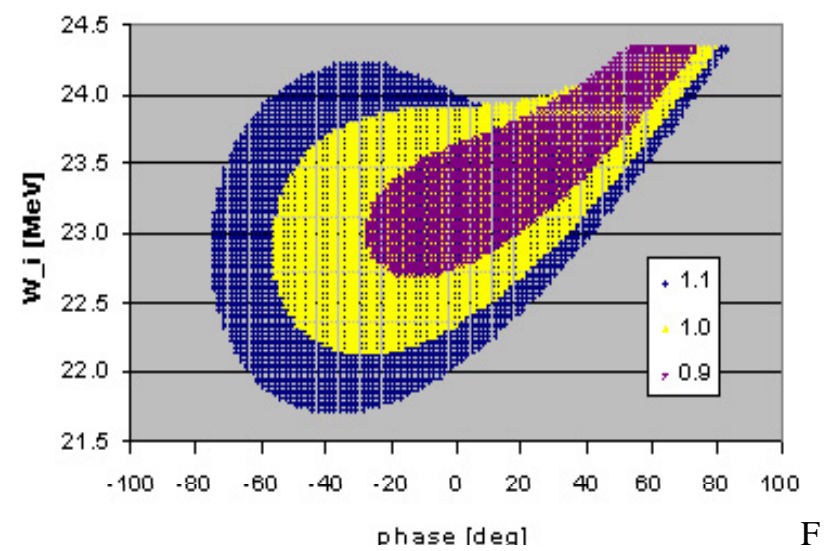

Figure 2. Longitudinal acceptance of DTL tank 3.

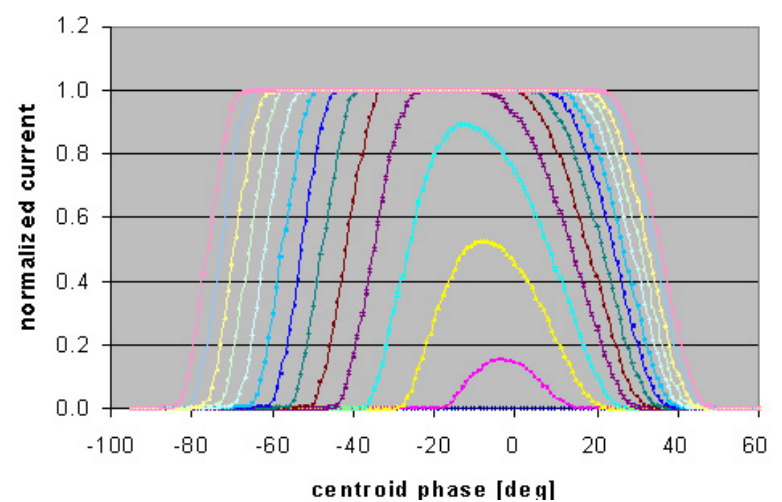

Figure 3. Traditional phase-scan plots for tank 3.

Figure 4 shows the composite energy spectrum that would be generated during the phase scan. Inserting a degrader with an energy range of $38 \mathrm{MeV}$, ahead of the
Faraday cup, effectively eliminates all unaccelerated beam from the beam current measurement.

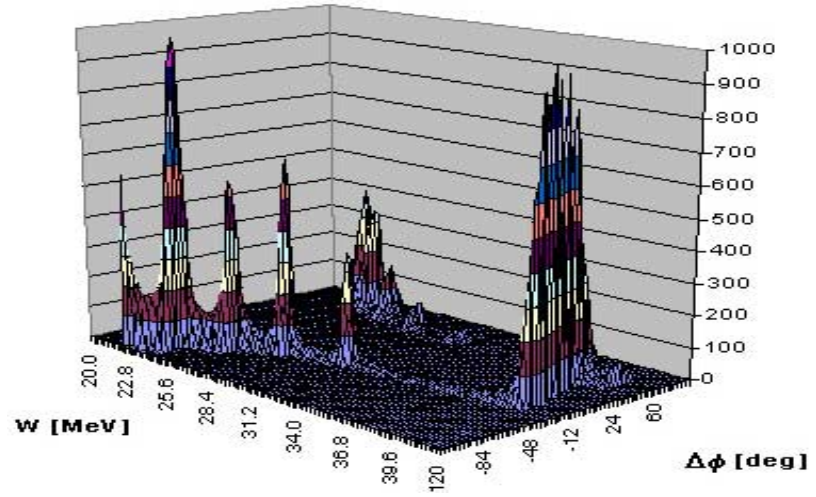

Figure 4. Relative transmitted beam current through tank 3 vs. $\phi_{\text {initial }}$ and $\mathrm{W}_{\text {initial }}$.

A second and third type of phase scan will be needed in some tanks in which we will measure either the beam's arrival time or its relative energy at the tank exit as a function of the tank's phase and amplitude. Relative energy measurements are made by time-of-flight (TOF) technique using the same BPMs described above for beam steering. Figure 5 shows a set TOF phase scans for tank 2 . On the vertical axis we plot the phase difference between the two BPMs in tank 3 . The different curves correspond to field amplitudes ranging from $98-106 \%$ of the design. value. In this case the total longitudinal phase advance is close to a multiple of $\pi$. For tanks where the phase advance is close to an odd multiple of $\pi / 2$, we measure the arrival time of the beam at a BPM in the next tank.

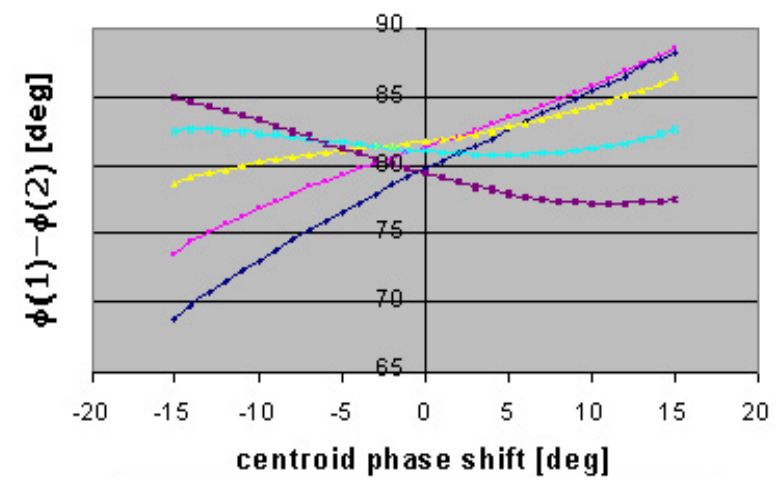

Figure 5. Phase scan of tank 2 using time-of-flight technique.

\section{COUPLED-CAVITY LINAC}

The CCL is comprised of $4 \mathrm{rf}$ modules containing 96 accelerating cells each. Each module is an assembly of twelve 8-cell segments resonantly coupled together via 3cell bridge couplers. Each module is excited via waveguide irises located in two of the eleven bridge couplers. The rf power from a single 5-MW klystron splits once to drive each module.

The CCL contains a total of fifty quadrupole lenses located between segments in an FD lattice of length $13-\beta \lambda_{805}$. The quadrupole gradients ramp down to match 
the beam from the strongly focused DTL lattice into the more weakly focused SRF lattice. Likewise, the cavity phase and amplitude are ramped in the first and last modules to match the $\mathrm{k}_{10}$ of the DTL and SRF linacs respectively.

Diagnostics, which are located adjacent to the quadrupoles, include $16 \mathrm{BPMs}, 8$ wire scanner profile monitors, 2 BCMs and 2 Faraday cups. Each quadrupole has dipole windings in the focusing plane, of which only a subset will be energized. The beam will be steered through the CCL using the least-squares steering algorithm [2] which makes use of multiple dipoles and BPMs to minimize beam excursions rather than occasionally placing the beam on the axis using dipole and BPM pairs.

The transverse match will be adjusted by observing envelope oscillations using wire scanners. A combination of TOF phase scans and $\Delta t$ [3] measurements will determine the phase and amplitude set points for each $\mathrm{rf}$ module.

Following the CCL, a 2.35-m-long drift contains differential pumping stations and an electrostatic precipitator to isolate the room-temperature linac from the ultra-clean high vacuum of the SRF linac. Special attention has been given to the design of the final CCL module to compensate this break in the periodicity of the transverse and longitudinal lattice. An insertable beam stop, located in this region, will be used when commissioning of the room-temperature linac at very low power beam.

\section{SUPERCONDUCTING LINAC}

The SRF linac has two sections. The low- $\beta$ section, with 33 6-cell cavities, accelerates the beam to $394 \mathrm{MeV}$. The high- $\beta$ section, with 48 6-cell cavities, accelerates the beam to $1 \mathrm{GeV}$. The low- $\beta$ section $(\beta=.61)$ has 11 cryomodules containing 3 cavities each, and the high- $\beta$ section ( $\beta=.81$ ) has 12 cryomodules with 4 cavities each. There is unsubscribed space in the tunnel to install 9 more high- $\beta$ cryostats, potentially allowing us to extending the present design to a beam energy of $\sim 1.5 \mathrm{GeV}$.

The SRF linac design philosophy has changed little since last reported [4]. However, it is now believed that higher accelerating fields will be achieved in the high- $\beta$ cavities. Figure 6 shows the expected distribution of the peak surface electric fields, $E_{\max }$, for the SRF cavities. The project schedule precludes any sorting of the cavities to optimize the linac design so the reference design assumes the mean values of these distributions. Figure 7 shows the design values of a) $E_{\max }$, b) $E_{0}$, the average axial accelerating field, and c) $\mathrm{E}_{0} \mathrm{~T}$, where $\mathrm{T}$ is the transit-time factor.

Given the cavity field, the number of cavities, and the final energy leaves only the cavity phase to be determined. The design phase, $\phi_{\text {design }}=-20.5^{\circ}$ and $-19.5^{\circ}$ in the low- and high- $\beta$ sections, respectively, meets the design requirements and provides a smooth match at the CCL: $\beta_{1}$ and $\beta_{1}: \beta_{2}$ lattice transitions. These relatively small phase angles provide high energy gain, but they reduce the longitudinal focusing and result in a small longitudinal acceptance. Error studies will determine if this choice causes problems.

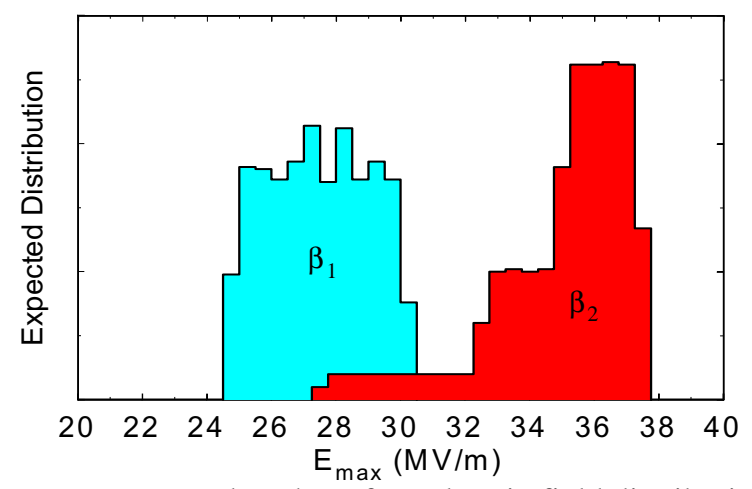

Figure 6. Expected peak surface electric field distribution in the SRF cavities.

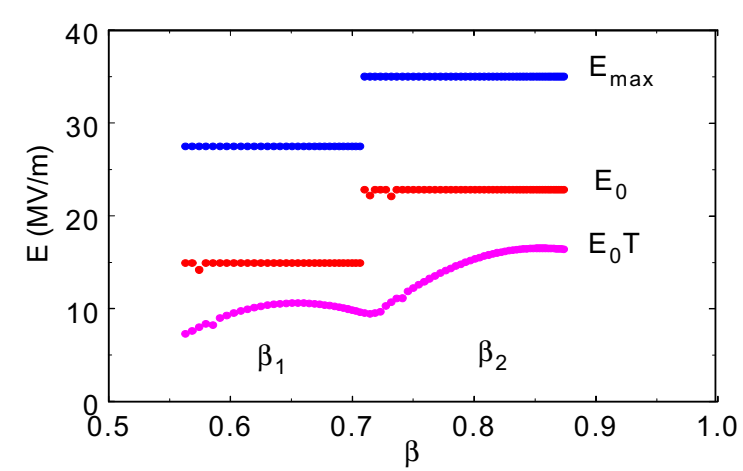

Figure 7. Electric field profiles in the SRF reference design.

Each SRF cavity is powered by a single $550 \mathrm{~kW}$ klystron. The lower curve in Fig. 8 shows the power that will be transferred to the beam in each cavity in the reference design. The upper curve shows the expected power demand for each klystron including a control margin. The low- $\beta$ cavities require a control margin of $33 \%$ of the beam power to meet the phase and amplitude stability requirements of $\pm 0.5 \%$ and $\pm 0.5^{\circ}$. We have reserved a $40 \%$ control margin for the higher-field high- $\beta$ cavities where the Lorentz-force detuning is expected to be $27 \%$ higher.

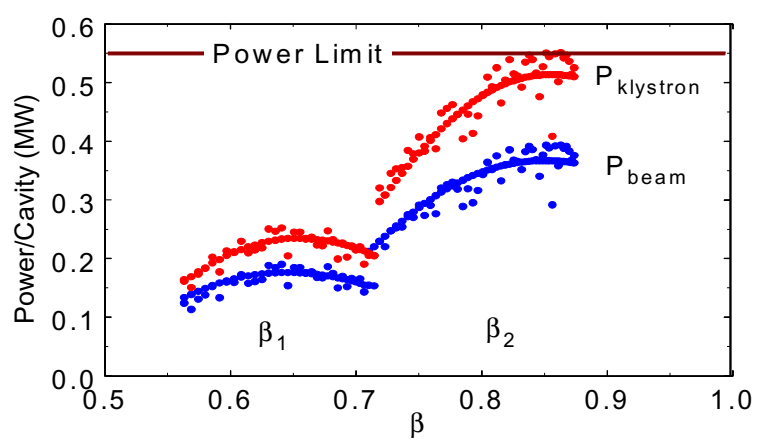

Figure 8 . RF and beam power per cavity. 
During cavity conditioning in the linac tunnel, we will measure the maximum achievable accelerating field, $\mathrm{E}_{0}$, of each cavity. The corresponding phase set points, $\phi_{\text {operating, }}$, will preserve the longitudinal phase advance, $\sigma_{01}$ of the reference design. This combination of $\mathrm{E}_{0}$ and $\phi_{\text {operating }}$ does not necessarily maintain the design accelerating rate. As a consequence, the transverse and longitudinal beam dynamics are expected to closely follow the reference design, but the final energy, $\mathrm{W}_{\text {final }}$, will not be known until all of the cavities are installed and their operating values determined. The scattered points in Fig. 8 show one set of beam and rf powers for cavity performances randomly sampled from the expected values in Fig. 6. Figure 9 shows the expected $\mathrm{W}_{\text {final }}$, which depends on the actual performance achieved in all the cavities.

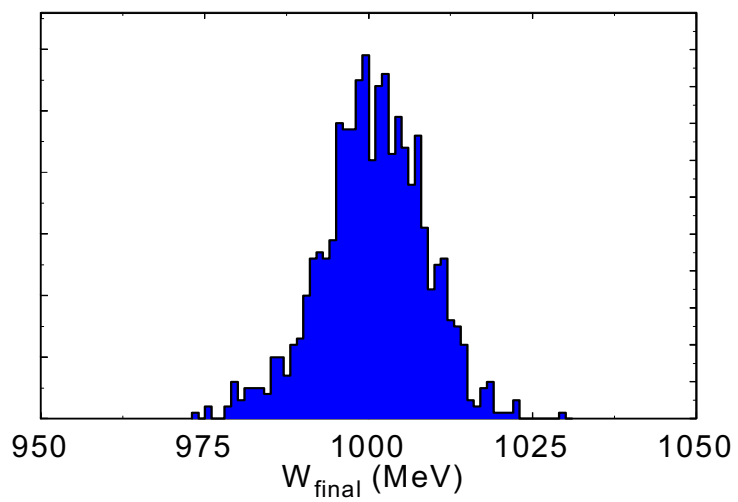

Figure 9. Expected final beam energy.

While we plan to take full advantage of the maximum achievable cavity fields to reach $1 \mathrm{GeV}$, the available rf power will limit the average beam current during a macropulse to $\sim 26 \mathrm{~mA}$. Taking into account the chopping duty factor, the peak beam current cannot exceed $\sim 38 \mathrm{~mA}$ assuming that some of the high performance cavities are located near the end of the linac. The average beam current at $6 \%$ duty factor will be $\sim 1.5 \mathrm{~mA}$.

Quadrupole doublets, located in the warm regions between cryostats, provide the transverse focusing. Each quadrupole has steering windings in the focusing plane. Diagnostics, located between each quadrupole pair, include a BPM and a wire-scanner profile monitor. Steering will be implemented using the least-squares technique using a subset of the dipoles. Transverse matching will be based on minimization of beam envelope oscillations as measured by the wire scanners.

RF pickup probes will be calibrated using single fullcurrent beam pulses to excite each cavity sequentially. The fields can be accurately predicted by numerical simulations [5]. This calibration relies on accurate measurements of the beam energy and current. A TOF measurement will determine the energy and the current will be measured using BCMs located at the entrance and exit of the SRF linac. The full calibration beam pulse must be transported to the linac beam dump to avoid damaging the linac.

\section{BEAM-DYNAMICS DESIGN}

We have selected focusing lattice parameters to avoid structure resonances throughout the linac. The zerocurrent phase advance per period, $\sigma_{0 \mathrm{t}}$ and $\sigma_{01}$ never exceeds $90^{\circ}$ as shown in Fig. 10. In the high- $\beta$ section of the SRF linac, where the expected value of $E_{\max }=35$ $\mathrm{MV} / \mathrm{m}, \sigma_{01}$ would exceed $90^{\circ}$ if not for the low value of $\phi_{\text {design. }}$. We avoid the second order parametric resonance by adjusting the quadrupole gradients so that $\sigma_{0 \mathrm{t}}$ and $\sigma_{01}$ do not cross except in DTL tank 1 and CCL module 4 where matching considerations take precedence.

We make $\mathrm{k}_{\mathrm{t} 0}$ and $\mathrm{k}_{10}$, the phase advance per meter, continuous across all lattice transitions as shown in Fig. 11. This design feature minimizes the potential for mismatches and helps assure a current independent design.

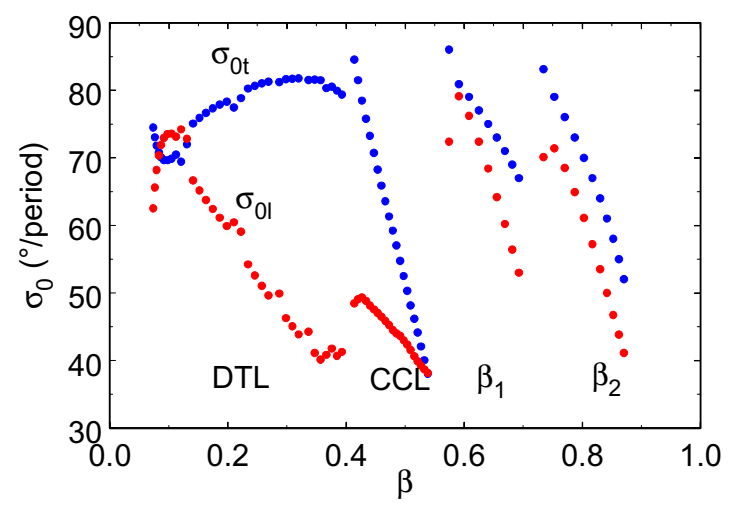

Figure 10. Zero-current transverse and longitudinal phase advance per period.

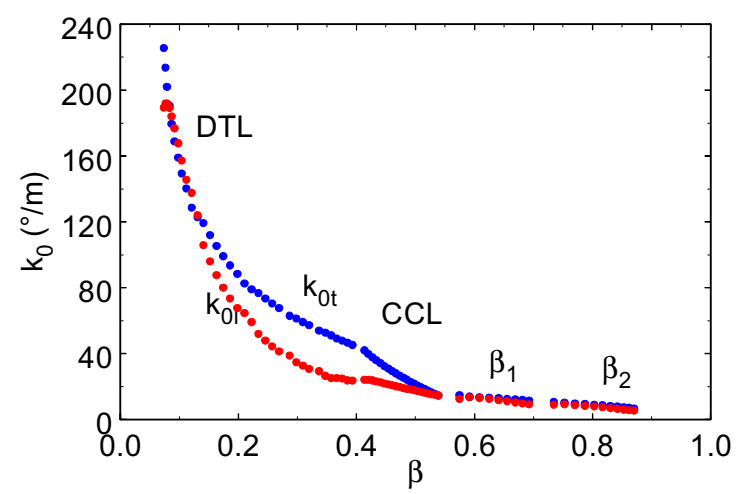

Figure 11. Zero-current transverse and longitudinal phase advance per meter.

Other sources of potential halo and emittance growth are resonant modes that can develop in the beam itself and cause energy transfer between planes. Anisotropy, $\mathrm{T}$, is a function of the tune and emittance ratios, and is believed to be the driving mechanism for such modes [6].

$$
\mathrm{T} \equiv \sigma_{\mathrm{l}} / \sigma_{\mathrm{t}} * \varepsilon_{\mathrm{l}} / \varepsilon_{\mathrm{t}}
$$

An equipartitioned design $(\mathrm{T}=1)$ typically avoids these resonances. Though the SNS design is not equipartitioned, the average emittance ratio is relatively low $\left(\varepsilon_{1} / \varepsilon_{\tau} \approx 1.2\right)$, thus limiting the potential for energy 
exchange. Emittance growth caused by beam resonances typically takes many betatron periods to develop. Table 2 shows that there is little time for such beam degradation to occur in the SNS linac.

Table 2. Linac temporal properties

\begin{tabular}{lccc}
\hline & DTL & CCL & SRF \\
\hline Acclerating gaps & 216 & 384 & 486 \\
Lattice periods & 37 & 24 & 23 \\
Betatron periods & 8 & 4 & 4 \\
Transit time (nsec) & 552 & 387 & 733 \\
\hline
\end{tabular}

Figure 12 is a theoretical resonance chart calculated for nominal SNS design parameters, which include a beam current of $52 \mathrm{~mA}$ and an emittance ratio of 1.2. The contoured peaks identify potential beam resonances. The shaded contours indicate the expected rates of emittance growth with $5 \%$ being the lowest value plotted. The tune trajectories of the SNS beam, superimposed on this diagram, correspond to a 52-mA beam.

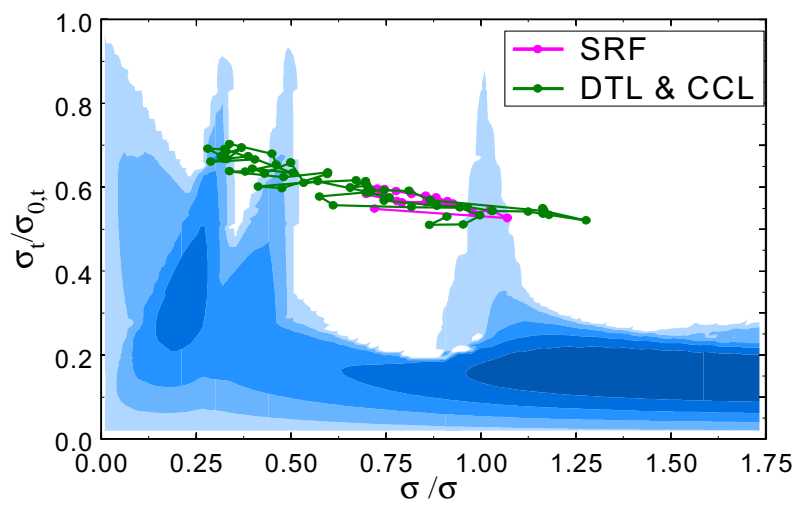

Figure 12. SNS tune trajectory superimposed on a theoretical resonance chart.

The two peaks on the left side of this chart represent weak resonances that take a long time to develop. The peak at a tune ratio $=1$ is a $3 \mathrm{rd}$ order resonance that starts from noise and requires 20-30 betatron periods to develop [7]. At $38 \mathrm{~mA}$ the tune depression will be less severe, causing the tune trajectories to move upward on the resonance chart, pushing the design further into the safe region.

\section{SIMULATION RESULTS}

To date all simulations have assumed a water-bag beam distribution at the entrance to the RFQ. Using this distribution we find that, without errors, the beam in the linac is well matched and experiences acceptable emittance growth as shown in Figs. 13 and 14. Error studies, using the same distribution, have shown that this design meets the specifications for beam emittance, beam loss, spatial stability, energy spread and energy stability. Further error studies are underway using a more realistic beam distribution derived from recent Front-End emittance measurements.

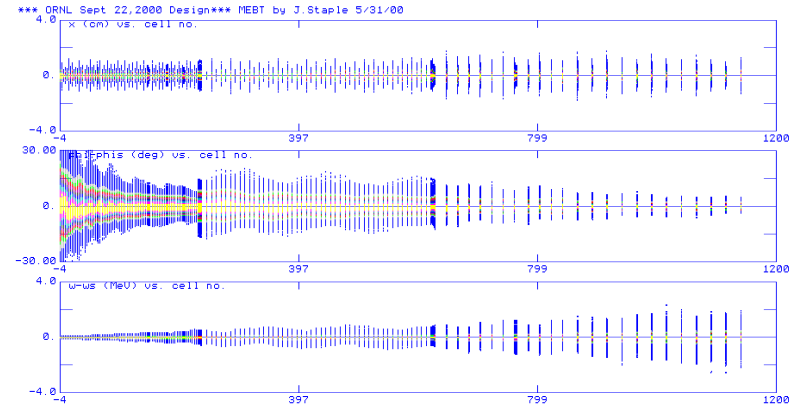

Figure 13. Horizontal (top), phase and energy (bottom) beam profiles.

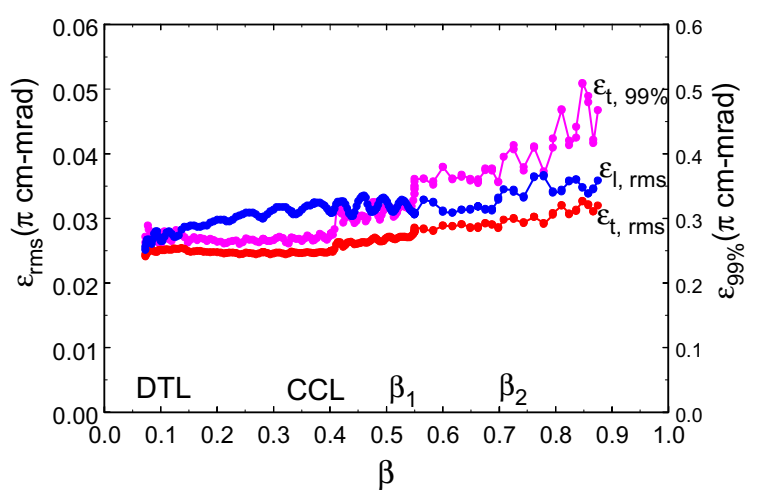

Figure 14. Beam emittance profile.

\section{CONCLUSION}

The SNS linac design has evolved significantly during the past 2 years into an affordable and efficient design. Comprehensive error studies, using more realistic beam distributions, are underway to quantify its expected performance. In addition, a complete set of tuning algorithms have yet to be developed for commissioning.

\section{REFERENCES}

[1] J. H. Billen, H. Takeda, and T. S. Bhatia, "Linac RF Structures for the Spallation Neutron Source," Proceedings of the 1999 Particle Accelerator Conference, p. 3585 (March 27 - April 2, 1999).

[2] K. Crandall, private communication

[3] K. Crandall et. al., "The $\Delta t$ Turn-On Proceedure," Proceedings of the Proton Linear Accelerator Conference, Oct. 10-13, 1972, Los Alamos, NM.

[4] J. Stovall et.al., "Superconducting linac for the SNS," Proceedings of the 2000 International Linac Conference, p. 605 (August 21-25, 2000).

[5] L. M. Young, "A procedure to Set Phase and Amplitude of the RF in the SNS Linac's Superconducting Cavities," this conference.

[6] I. Hoffmann, J. Qiang and R. Ryne, Phys. Rev. Lett. 86, 2313 (2001).

[7] I. Hoffmann et. al., "Emittance Coupling in High Intensity Beams Applied to the SNS Linac," this conference. 